\title{
Trabajo EN EQUipo y DesarRollo Del PENSAMiento CRÍTICO EN LA FORMACIÓN DOCENTE EN LA Universidad Nacional Daniel Alcides Carrión, Pasco, Perú
}

\section{TEAM WORK AND DEVELOPMENT OF CRITICAL THINKING IN TEACHER TRAINING AT THE DANIEL AlCIDES Carrion National University, Pasco, Peru}

\author{
Julio César Carhuaricra Meza \\ Universidad Nacional Daniel Alcides Carrión, Edificio Estatal No 4 , San Juan Pampa, Cerro de \\ Pasco, Pasco
}

\begin{abstract}
RESUMEN
El trabajo en equipo (T. E.) y el desarrollo de pensamiento crítico (P. C.) son competencias que debemos promover en los estudiantes universitarios, como lo plantea el proyecto Tuning de América Latina. Guiado por tal demanda, nos propusimos determinar el nivel de influencia del primero (T. E.) sobre el segundo (P. C.) en el contexto de la formación docente. Para ello, se ha elegido el tipo de estudio correlativo y explicativo, y respecto al diseño, se ha elegido el cuasiexperimental, con tratamientos múltiples y evaluaciones de pre test y post test. Asimismo, se ha considerado dos grupos: experimental y control. El universo lo constituyeron 753 alumnos y la muestra 60 alumnos. Las técnicas aplicadas fueron: para la muestra, no probabilística con población finita; para la recolección de datos, la encuesta y análisis documentario. La principal conclusión a la que ha llegado la investigación es que el trabajo en equipo influye favorablemente en el desarrollo del pensamiento crítico en la formación docente.
\end{abstract}

Descriptores: trabajo en equipo, desarrollo del pensamiento critico y formación docente

\begin{abstract}
Working in teams and the development of critical thinking are capabilities that we must promote in university students, as proposed by the Latin America Tuning project. Guided by such demand we set out to determine the influence of the first (T.E.) upon the second one (P.C.) in the context of teacher training. We have selected a correlative and explanatory study type, the design chosen is the quasi-experimental, with multiple treatments with evaluations of pre test and post test. At the same time, we considered two groups: experimental and control. The universe was constituted of 753 alumni and the sample of 60 alumni. The applied techniques were: for the samples, not probabilistic with finite population; for the data collection survey and documentary analysis. The main conclusion reached by this investigation is that teamwork influences favorably in the development of critical thinking in the teacher training.
\end{abstract}

Keywords: work team, development of critical thinking and training teachers 


\section{INTRODUCCIÓN}

El desarrollo de las competencias interpersonales, trabajo en equipo y el pensamiento crítico, son necesarios en los tiempos actuales (Informe Final, Proyecto Tuning-América Latina 2004-2007). Este hecho nos ha motivado a investigar el desarrollo de dichas competencias en el contexto de la formación docente, nivel de educación secundaria, en la Facultad de Ciencias de la Educación y Comunicación Social, Universidad Nacional Daniel Alcides Carrión, Pasco, Perú.

Se entiende por trabajo en equipo "[a] una serie de estrategias, procedimientos y metodologías que utilizan un grupo humano para lograr las metas propuestas, asumir el papel gerencial de parte del coordinador, asignar prioridades, dedicarse a los miembros del equipo y valorar las contribuciones" [1].

Además, por pensamiento crítico se comprende "[e]se modo de pensar sobre cualquier tema, contenido o problema en el cual el pensante mejora la calidad de su pensamiento al apoderarse de las estructuras inherentes al acto de pensar y al someterlas a estándares intelectuales" [2]. Para fines del estudio, se ha tomado en cuenta tres niveles: literal, inferencial y crítico.

El problema principal que pretendió contestar el estudio es: ¿De qué manera el trabajo en equipo influye sobre el desarrollo del pensamiento crítico en la formación docente de la Universidad Nacional Daniel Alcides Carrión de Cerro de Pasco, Perú?

Por consiguiente, el objetivo general fue determinar la influencia del trabajo en equipo sobre el desarrollo del pensamiento crítico en la formación docente de la Universidad Nacional Daniel Alcides Carrión de Cerro de Pasco, Perú.

La hipótesis general que ha orientado el estudio fue: la aplicación de trabajo en equipo influye favorablemente en el desarrollo del pensamiento crítico en la formación docente de la Universidad Nacional Daniel Alcides Carrión de Cerro de Pasco, Perú

Y la principal conclusión a la que se ha arribado es que el trabajo en equipo expresado en la valoración de sus integrantes influye favorablemente en el desarrollo del pensamiento crítico, principalmente en el nivel inferencial.

\section{MÉTODO EXPERIMENTAL}

El estudio fue de tipo correlacional y de nivel explicativo.

Se utilizó el diseño cuasi experimental con dos grupos, y tratamientos múltiples con pre test $\mathrm{y}$ post test. Ello nos ayudó a determinar la relación de influencia de la primera variable, trabajo en equipo, sobre la segunda variable, desarrollo del pensamiento crítico.

El universo de estudio lo constituyeron todos los estudiantes de la Escuela de Formación Profesional de Educación Secundaria (763 Estudiantes). La muestra representativa la constituyeron 60 estudiantes de los semestres VIII (30 individuos grupo control, que llevaron el curso de Prácticas Preprofesionales) y VI (30 individuos - grupo experimental que llevaron el curso de Didáctica de Biología y Química), ambos grupos pertenecientes a la especialidad de Biología y Química.

En cuanto a las técnicas e instrumentos de investigación, se aplicaron las siguientes: a) De muestreo no probabilístico con población finita; b) De recolección de datos: encuestas, cuyo instrumento fue el cuestionario para estudiantes, que contó con 28 reactivos, y cuya primera parte enfatiza el T. E. y segunda parte el P. C. y el análisis documentario (en este caso el instrumento fue la guía de análisis documentario); c) De experimentación: se desarrollaron las clases mediante trabajos en equipo organizados por el docente de clase en cinco grupos de alumnos para la asignatura de Prácticas Preprofesionales del VIII Semestre (grupo experimental). El mismo docente también desarrolló la asignatura de Didáctica de Biología y Química con alumnos del VI semestre. En ella se utilizaba como estrategia didáctica tradicional el énfasis en la exposición (grupo control). Y d) Para el procesamiento y análisis de datos: preparación de datos, revisión, corrección y verificación de las respuestas; si son legibles, completas y coherentes, y categorización de las respuestas. Asimismo, la codificación y tabulación de datos: la primera asignó 
números a las respuestas y la segunda presentó tablas de distribución de frecuencias en función a las categorías de las variables, que luego fueron interpretadas.

Tabla 1: Diseño cuasi experimental con dos grupos, tratamientos múltiples, con pre test y post test.

\begin{tabular}{|l|l|l|l|l|l|l|l|}
\hline GRUPOS & $\mathrm{V}_{2}$ & $\mathrm{~V}_{1}$ & $\mathrm{~V}_{2}$ & $\mathrm{~V}_{1}$ & $\mathrm{~V}_{2}$ & $\mathrm{~V}_{1}$ & $\mathrm{~V}_{2}$ \\
\hline Experimental & $\mathrm{O}_{\mathrm{i}}$ & $\mathrm{X}_{1}$ & & $\mathrm{X}_{2}$ & & $\mathrm{X}_{3}$ & $\mathrm{O}_{\mathrm{p}}$ \\
\hline Control & $\mathrm{Oi}$ & & & & & & $\mathrm{O}_{\mathrm{p}}$ \\
\hline
\end{tabular}

Grupo experimental $=30$ estudiantes del VIII semestre, sección "C", que llevaron la asignatura de Prácticas Preprofesionales II-B de la especialidad de Biología y Química (Semestre: 2009-B)

Grupo Control $=30$ estudiantes del VI semestre, sección $\mathrm{C}$, que llevaron el curso de Didáctica de Biología y Química (Semestre: 2009-B) o

$\mathrm{V}_{1}=$ Primera variable: trabajo en equipo.

$\mathrm{V}_{2}=$ Segunda variable: desarrollo del pensamiento crítico.

Oi $=$ Observación inicial $=$ Pre Test .

$\mathrm{X}_{1}, \mathrm{X}_{2}, \mathrm{X}_{3,}=$ Tratamientos experimentales.

Op = Observación terminal $=$ Post Test .

\section{RESULTADOS}

\section{Valoración de las ideas en el trabajo en equipo}

En el grupo experimental: en el pre test, el 100\% afirmó que sí valora las ideas de los miembros en el trabajo en equipo. En el post test, el 100\% también afirmó que sí. De manera que se valora y se reconoce las ideas en el trabajo en equipo.

En el grupo control: en el pre test, el 90\% dijo que sí valora las ideas de los miembros en el trabajo en equipo y el $10 \%$ que no. En el post test, el $97 \%$ afirmó que sí y el 3\% que no. Por lo tanto, se comprueba que también en este grupo prima la valoración y reconocimiento de las ideas de parte de los miembros del equipo.

Se concluye que en el trabajo en equipo se valora y reconocen las ideas.
Tabla 2: Valoración de las ideas en el trabajo en equipo.

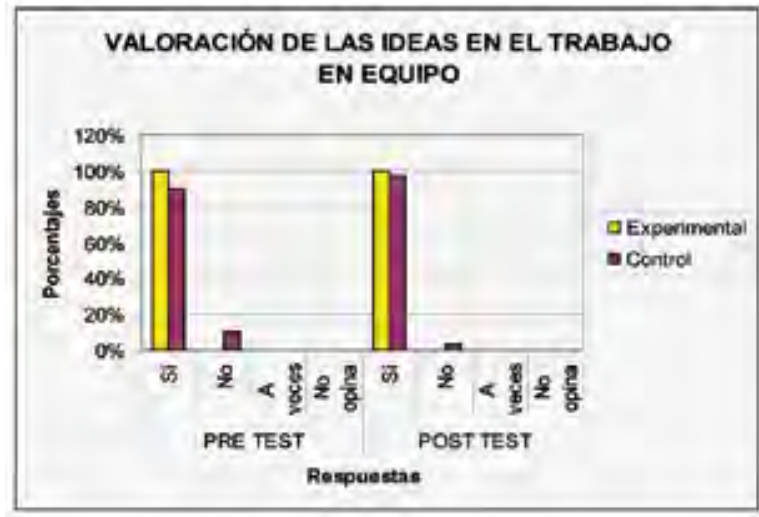

Reconocimiento público en el trabajo en equipo

En el grupo experimental: en el pre test, el 54\% afirmó que a veces reconoce públicamente el aporte de los miembros en el trabajo en equipo; el 33\% que sí reconoce, y el $13 \%$ que no. En el post test, el $70 \%$ afirmó que sí, el $17 \%$ que no y el $13 \%$ a veces. Por ende, en el trabajo en equipo se practica el reconocimiento público.

En el grupo control: en el pre test, el 56\% dijo reconocer a veces públicamente el aporte de los miembros en el trabajo en equipo, el $27 \%$ afirmó que sí, y el $17 \%$ que no. En el post test, el $56 \%$ a veces, el $24 \%$ sí y el $20 \%$ no. Por lo tanto, a veces se realiza el reconocimiento público por el aporte de los miembros en el trabajo en equipo.

Por consiguiente, es evidente el reconocimiento público en el trabajo en equipo.

Tabla 3: Reconocimiento público en el trabajo en equipo.

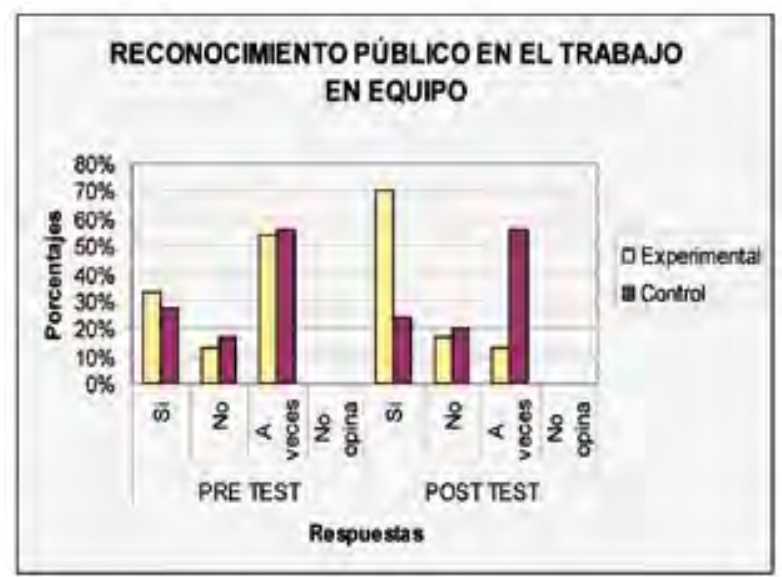


Valoración de los pequeños aportes en el trabajo en equipo

En el grupo experimental: en el pre test, el $73 \%$ afirmó que sí valora los pequeños aportes de los miembros en el trabajo en equipo y el $27 \%$ que a veces. En el post test, el $83 \%$ sí, $10 \%$ no y $7 \%$ a veces. Se demuestra que en el trabajo en equipo valoran los pequeńos aportes de sus integrantes.

En el grupo control: en el pre test, el 77\% dijo que sí valora los pequeños aportes de los miembros en el trabajo en equipo, el $20 \%$ que a veces y el $3 \%$ que no. En el post test, el $73 \%$ dijo que sí, el $17 \%$ a veces y el $7 \%$ no. Por lo tanto, en este grupo también se presenta en forma significativa la valoración de los pequeños aportes de los integrantes del trabajo en equipo.

De manera que en el trabajo en equipo se valoran los pequeńos aportes de sus integrantes.

Tabla 4: Valoración de los pequeños aportes en el trabajo en equipo.

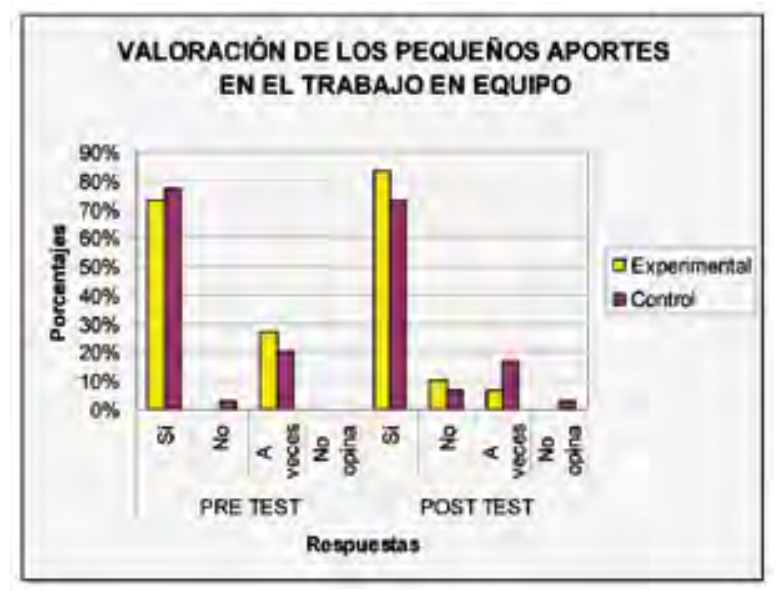

\section{Reconocimiento en el trabajo en equipo}

En el grupo experimental: en el pre test, el $87 \%$ de estudiantes afirmó que sí reconoce las contribuciones de los miembros en el trabajo en equipo y el 13\% que a veces. En el post test, el $97 \%$ sí y el $3 \%$ que no. Se evidencia que en el trabajo en equipo se promueve el reconocimiento por las tareas realizadas.

En el grupo control: en el pre test, el 77\% dijo que sí reconoce las contribuciones de los miembros en el trabajo en equipo, el 13\% que a veces y el 10\% que no En el post test, el $87 \%$ dijo que sí y el 13\% que no. En este grupo se hace evidente el reconocimiento por el trabajo realizado.

Entonces, en el trabajo en equipo se práctica el reconocimiento por las tareas que pueden realizar sus miembros.

Tabla 5: Reconocimiento en el trabajo en equipo.

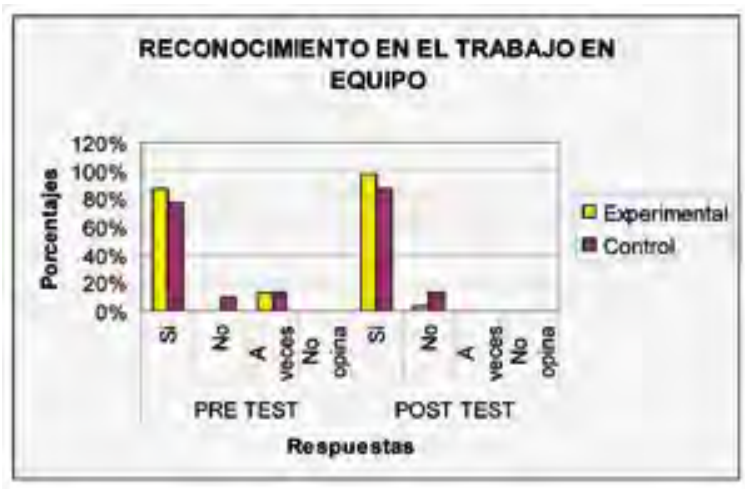

\section{Identificación de informaciones en un texto}

En el grupo experimental: en el pre test, el 53\% identificó correctamente las informaciones que utilizó el autor de un texto para sostener sus argumentos, el 27\% identificó incorrectamente y el $10 \%$ identificódeunamaneramedianamentecorrecta. En el post test, el 80\% identificó correctamente, el $17 \%$ de una manera medianamente correcta y el $3 \%$ de manera incorrecta. Se comprueba que en el trabajo en equipo se identifica correctamente las informaciones que utiliza el autor de un texto para defender sus argumentos.

En el grupo control: en el pre test, el 50\% identificó correctamente las informaciones que presenta el autor para sustentar sus argumentos, el 33\% identificó incorrectamente y el $17 \%$ de manera medianamente correcta. En el post test, el 60\% identificó correctamente, el 23\% incorrectamente y el $17 \%$ medianamente correcta. En consecuencia, en este grupo se mantiene altos porcentajes en la identificación correcta de las informaciones que utiliza un autor de un texto para defender sus argumentos.

Se afirma que el trabajo en equipo favorece la identificación de las informaciones que presenta el autor de un texto para sustentar sus argumentos. 
Tabla 6: Identificación de informaciones en un texto.

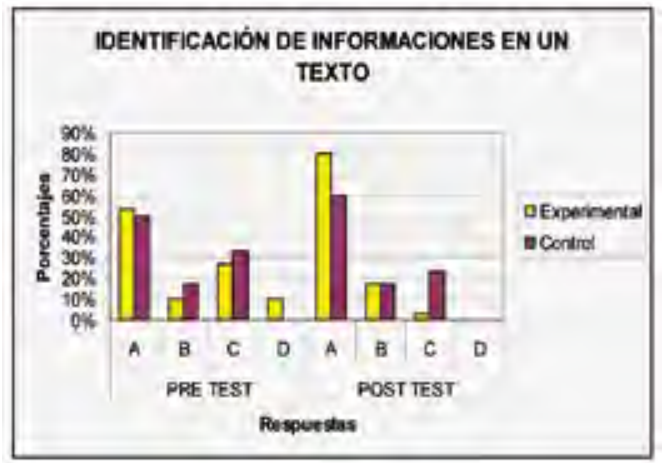

\section{Identificación de los principales conceptos en un texto}

En el grupo experimental: en el pre test, el 53\% identificó de manera medianamente correcta los principales conceptos que el autor expone en un texto, el 34\% identificó incorrectamente y el 13\% identificó correctamente. En el post test, el 70\% identificó correctamente, el 23\% medianamente correcta y el 7\% incorrecto. En suma, el trabajo en equipo favorece la identificación de los principales conceptos que expresa el autor de un texto.

En el grupo control: en el pre test, el 57\% identificó de manera medianamente correcta los principales conceptos que el autor utiliza al escribir un texto, el $23 \%$ identificó correctamente y el $20 \%$ incorrectamente. En el post test, el 60\% identificó de manera medianamente correcta, el $27 \%$ correctamente y el 13\% incorrectamente. En suma, se hace explícito que el trabajo en equipo favorece la identificación de los principales conceptos que el autor expresa en un texto.

En conclusión, en ambos grupos de estudio prevalece la tendencia de que el trabajo en equipo favorece la identificación de los principales conceptos que el autor presenta en un texto

Tabla 7: Identificación de los principales conceptos en un texto.

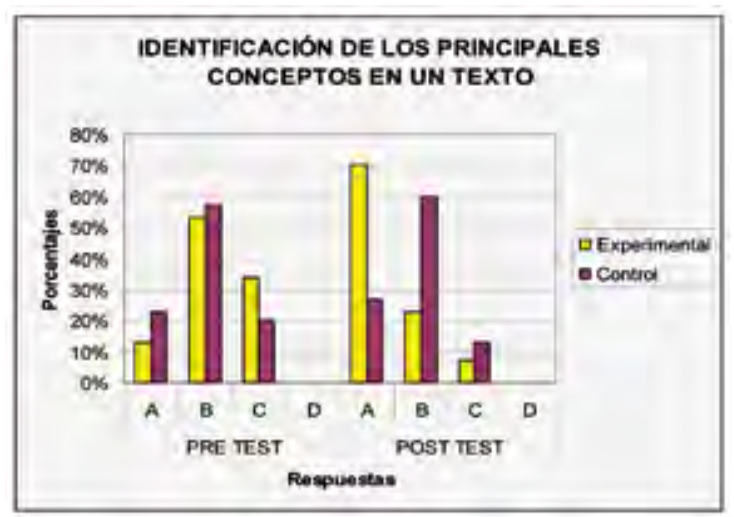

\section{Identificación de conclusiones en un texto}

En el grupo experimental: en el pre test, el $40 \%$ identificó correctamente las conclusiones expresadas en el texto de lectura, el 27\% identificó de manera medianamente correcta, el 20\% incorrectamente y el 13\% no opinó. En el post test, el 63\% identificó correctamente, el $27 \%$ medianamente correcta y el $10 \%$ incorrectamente. Por ende, el trabajo en equipo favorece la identificación de las conclusiones expresadas en un texto.

En el grupo control: en el pre test, el 43\% identificó correctamente las conclusiones presentes en el texto delectura, el 30\% identificó de manera medianamente correcta y el $27 \%$ de manera incorrecta. En el post test, el 40\% identificó correctamente, el 33\% medianamente correcta y el $27 \%$ incorrectamente. Se hace explícito que el trabajo individual y en equipo ayudan a la identificación de las conclusiones expresadas en un texto.

En conclusión, como se evidencia en los grupos de estudio, el trabajo en equipo favorece la identificación de las principales conclusiones presentes en un texto.

Tabla 8: Identificación de conclusiones en un texto.

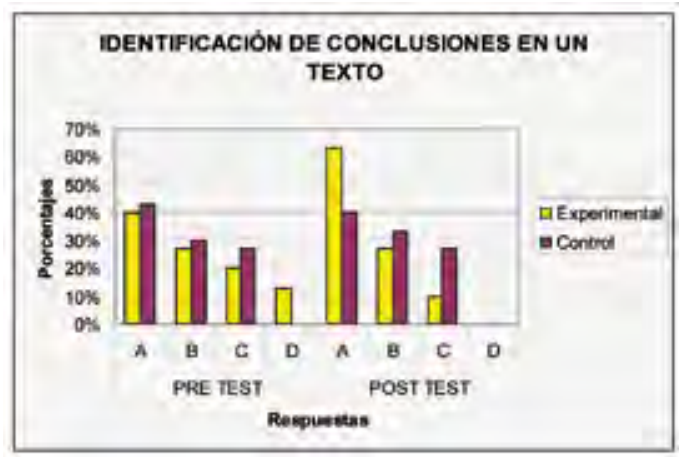

\section{DISCUSIÓN}

Como podemos ver, el estudio tiene como base la propuesta de Fischman [1] referente al trabajo en equipo, tomando básicamente la definición operacional y los indicadores. Asimismo, se considera los aportes de Borrell [3] en torno a la planificación, ejecución y evaluación de los trabajos en equipo. No obstante, los estudiosos mencionados nos explican la variable (T. E.) en 
contextos empresariales, mas no así en contextos educativos a nivel universitario; por ello creemos que la experiencia que presentamos es significativa.

Por otro lado, referente al pensamiento crítico, se toma en cuenta la visión de Paul y Elder [4], que dan mucha importancia a la dimensión lógica presente en los textos, los mismos que también desarrollan conceptos y herramientas para el desarrollo del P. C. Además, en este mismo horizonte, reconocemos el valioso aporte de Hawes [5] que nos habla del pensamiento crítico en la formación universitaria de Chile, Universidad de Talca.

Considerando todo lo anterior e invitando a profundizar la investigación, es importante validar la propuesta de Ennis [2] que puntualiza doce categorías de análisis del pensamiento crítico y tres dimensiones: dimensión lógica, dimensión criterial y dimensión pragmática.

\section{CONCLUSIÓN}

Se ha comprobado la influencia favorable del trabajo en equipo expresado en la valoración de sus integrantes sobre el desarrollo del pensamiento crítico, específicamente en el nivel inferencial. Todo ello en el contexto de la formación docente.

\section{AGRADECIMIENTOS}

Un reconocimiento especial a los directivos, docentes y alumnos de la especialidad de Biología y Química de la Escuela de Formación Profesional de Educación Secundaria, Facultad de Ciencias de la Educación, Comunicación y Derecho, de la Universidad Nacional Daniel Alcides Carrión, en la ciudad de Cerro de Pasco, Perú.

\section{REFERENCIAS}

[1] S. Fishman, El espejo del líder, UPC - El Comercio, Lima, octubre, 2000.

[2] Ministerio de Educación del Perú, Guía para el desarrollo del pensamiento crítico, Archivo Adobe Acrobat Document 5,14 MB en www. ministeriodeeducación.com, 2004.

[3] F. Borrell, Cómo Trabajar en equipo, Deusto y diario El Comercio, Lima, 2003.

[4] R. Paul y L. Elder, Cómo leer un párrafo y más allá de éste, Fundación para el pensamiento crítico, archivo en pdf, en www.criticalthinking.org, 2003.

[5] G. Hawes, Pensamiento crítico en la formación universitaria, Instituto de Investigación y Desarrollo Educacional de la Universidad de Talca, Chile, 2006.

E-mail: juliocesar1506@hotmail.com 